\title{
Analyis of Single and Networked Auctions
}

\author{
EROL GELENBE \\ Imperial College London
}

\begin{abstract}
Web based computerised auctions are increasingly present in the Internet. We can imagine that in the future this trend will actually be extended to situations where virtual buyer and seller agents will conduct automated transactions across the network, and that large sectors of the economy may be strucured in this manner. The purpose of this paper is to model automated bidders and sellers which interact through a network. We model the bidding process as a random arrival process while the price attained by a good is modelled as a discrete random variable. We obtain analytical solutions allowing us to compute the income from a single auction, or the income per unit time from a repeated sequence of auctions. A variety of single auction models are studied, including English and Vickrey auctions, and the income per unit time is derived as a function of other parameters including the rate of arrival of bids, the seller's decision time, the value of the good, and the "rest time" of the seller between successive auctions. We illustrate the results via numerical examples. We also introduce a model for networked auctions where bidders can circulate among a set of interconnected auctions which we call the "mobile bidder model (MBM)". We obtain an analytical solution for the MBM under the assumption,which we call the "active bidders assumption", that activities that are internal to an auction (bids and sales) are much more frequent than changes that occur in the number of bidders at each auction.
\end{abstract}

Categories and Subject Descriptors: C.2 [Computer Communication Networks]: Computer Networks; C.4 [Performance of Systems]: Analytical Models; G.3 [Stochastic Processes]: Queueing Systems

General Terms: Internet Technologies, E-Commere

Additional Key Words and Phrases: Automated Auctions, Autonomic Systems

\section{INTRODUCTION}

Auctions of different types have long been an important element of market based economics [Milgrom and Weber 1982] since the bidding and selling process can be formally specified and run in a timely fashion so that neither the bidders nor sellers are indefinitely held up. When a seller accepts a bid, it is implicitly rejecting other offers and making it known; the remaining bidders can then turn their attention to other opportunities to purchase a similar good. Very often the seller accepts a bid based on the fact that the corresponding offer is either the highest bid that

Author's address: Intelligent Systems and Networks Group, Department of Electrical and Electronic Engineering, Imperial College, London, SW7 2BT, UK. Email: e.gelenbe@imperial.ac.uk, Web: http://www.ee.ic.ac.uk/gelenbe. This research was undertaken as part of the ALADDIN (Autonomous Learning Agents for Decentralised Data and Information Systems) project and is jointly funded by a BAE Systems and EPSRC (Engineering and Physical Research Council) strategic partnership under Grant No. EP/C548051/1.

Permission to make digital/hard copy of all or part of this material without fee for personal or classroom use provided that the copies are not made or distributed for profit or commercial advantage, the ACM copyright/server notice, the title of the publication, and its date appear, and notice is given that copying is by permission of the ACM, Inc. To copy otherwise, to republish, to post on servers, or to redistribute to lists requires prior specific permission and/or a fee.

(C) 2006 ACM 0000-0000/2006/0000-0001 $\$ 5.00$ 
has been made, or in some cases it may choose one that is close to the highest bid in some reasonable sense, for instance when other factors are also to be taken into account.

Web based computerised auctions are increasingly present in the Internet. We can imagine that in the future this trend will be even more prevalent when virtual buyer and seller agents will conduct automated transactions across the world, so that large sectors of the economy may be strucured in this manner. The importance of auctions increases as much of the economy becomes virtual and network based, because through their formal algorithmic structure auctions are particularly amenable to Internet based implementations. Links between different markets, commodities or neighbouring markets can also lead to complex interconnected auctions and bidding strategies [Shehory ; 2002; David et al. 2005].

Although we naturally think of auctions as being related to economic activity, they are also used for decision making in resource allocation, for instance to request and receive network services or for physical resources in computer systems and networks such as buffer space or bandwidth. Auctions are an example of Computational Mechanisms [Das et al. 2003] which are studied as tools for the coordination of agent based systems.

In this paper we study auctions to which bidders' offers arrive sequentially at random time intervals. Successive bids are increasing in value, but the seller may not know the current market value of the good. The seller's problem is therefore to decide when it accepts a bid. Thus after each new bid, the seller may wait for a "decision delay or time" to determine whether to accept the offer. If a new bid arrives before that time expires, then the process is repeated for this new bid; at the end of the decision time, if a new bid has not arrived, the seller sells his good to the buyer who has so far made the most recent and therefore highest bid. After selling the good, the seller rests for a some time before initiating a new auction. This rest period can represent a technical delay in restarting an auction: in the case of a fully automated system everything would take place at "computer system times", i.e. milliseconds rather than human level times. In other cases the rest time may represent a delay in determining whether another good is available for sale. The case when this rest time is zero is also discussed (see Comment 2 in Section $2.2)$.

A related question which has received some attention is the "Secretary, or Sultan's Dowry, Problem" introduced by Martin Gardner in his column on mathematical recreations in the magazine Scientific American in 1960. The problem is to select the best candidate from a sequence of applicants, where the quality of the successive candidates are random variables. The decider has to select one candidate without the possibility of changing his/her mind, so that a candidate who has been turned down cannot be reconsidered, and once a candidate is selected or accepted the selection process stops and further candidates are not considered. The known optimal solution [Chow et al. 1964; Finch 2003] results in the recruitment of a candidate whose quality is close to the maximum, and the probability that it results in selecting the best candidate is obtained. In the auction models we study, successive bids have increasing values, contrary to the secretary problem where successive candidates have variable values. In the auction model although it is nice to sell a 
good for a high price, but it may be more important to take decisions so that the income per unit time is maximised, contrary to the secretary problem where the person that is hired stays "forever" and therefore must have the highest possible value. Analysis of auctions where bidders arrive, have a private valuation, and depart if they have not been allocated the good can be found in [Hajiaghayi et al. 2004]. Another related model [Guo 2002] has bidders which arrive in discrete time steps, while the seller instantaneously accepts or declines a bid; in the latter case the bidder immediately disappears; it leads to an optimal stopping time problem and it is solved analytically.

The purpose of this paper is to model automated bidders and sellers which interact through a network. We model the bidding process as a random arrival process while the price attained by a good is modelled as a discrete random variable. We obtain analytical solutions allowing us to compute the income from a single auction, or the income per unit time from a repeated sequence of auctions. A variety of single auction models are studied, including English and Vickrey auctions, and the income per unit time is derived as a function of other parameters including the rate of arrival of bids, the seller's decision time, the value of the good, and the "rest time" of the seller between successive auctions. We illustrate the results via numerical examples. We also introduce a model for networked auctions where bidders can circulate among a set of interconnected auctions which we call the "mobile bidder model (MBM)". We obtain an analytical solution for the MBM under the assumption, which we call the "active bidders assumption", that activities that are internal to an auction (bids and sales) are much more frequent than changes that occur in the number of bidders at each auction.

Of course, human buyers and sellers in auction systems are rational agents who pursue some possibly self-interested agenda; for instance one would not expect that a seller's objective is to minimise his or her income and give goods at the lowest possible price that just covers the manufacturing cost of a good, although this may indeed be an objective in certain contexts. Similarly, we would expect bidders to find ways to drive away the conmpetition (for instance by moving the price to a level where competitors remove themselves from the competition), but yet they would try to purchase a good at the lowest possible price. This kind of "rationality" would also carry over to automated buyers and sellers where programmed computer based rules would try to mimic the self-interest or rationality of the agents.

In this paper we do not try to model the internal workings of the agents through the complex behavioural rules or reactions that they may have, though we do not exclude such representations in our future work, for instance through the use of machine learning or neural networks which may be used by bidders and sellers to pursue specific rational objectives. However we do include some elements of rational or self-interested behaviour, although this is limited to some collective, i.e. common to all behaviours, rather than individual properties.

The rate at which bidders make their bids is itself a representation of how enthusiastic the community of bidders may be. The notion of "value" or valuation, discussed in Section 2 and used throughout the paper, is one representation of this rationality, since the assumption is that when the most recent bid has attained or exceeded the value $V$, then another bidder will not make a new bid; we assume 
that $V$ is a random variable with a common distribution function. Another element that models some level of rationality among bidders is the "state dependent bidding rate" introduced in Section 2.6, where we show how the model can be analysed when the rate at which bids arrive depends on the level that the most recent bid has attained. This can be applied to the case, for instance, where bids arrive at a slower rate as the value of the most recent bid increases, although it may be used for any dependence between the bidding rate and the attained bid price. Throughout this work we consider that the time it takes the seller to take a decision to sell is a the main tool it has to express its rationality; here again, in future work we plan to investigate adaptive approaches based on on-line observation of other parameters of the market.

In the following two sections we will study single auction models. The fourth section of the paper is devoted to networked auctions where bidders are allowed to move among $N$ auctions that sell one or several types of goods. The last section presents conclusions and some open questions.

\section{AN AUCTION WITH UNIT INCREMENTS}

We first analyse an auction in which bidders increase their offers in unit increments. We assume that bids arrive to the seller one at a time, according to a Poisson process of rate $\lambda$ so that the average time that will elapse between successive bids is $\lambda^{-1}$. If a bid is not accepted by the seller, then the next bid will increment the value of the offer by 1 as long as the value $V$ of the good for the buyers has not been reached. However the buyers will stop bidding and incrementing the offer when the most recent bid has reached the value $V$ that the bidders associate with it.

We assume that the seller does not know the value $V$ that buyers associate with the good, or that it makes no use of historical data about it. In any case our asssumption is that the seller just relies on its ongoing observation about whether a new bid arrives. Thus after each bid, the seller waits for some random "decision time" to determine whether to accept the offer. If a new bid arrives before that time expires, then the process is repeated for this new bid. However if a new bid does not arrive before this time expires, then the seller accepts the current bid. Of course, if the seller accepts the offer too quickly, then the price he will be getting may be low with respect to the price that he would have gotten had he been more patient. On the other hand, if the buyer waits a long time before accepting an offer he may ultimately get the highest possible price (which is $V$ ), but at the expense of wasting a lot of time. Note that if bidders are parsimonious and only bid up to some price $R=V-h$, where $h \geq 0$ and represents the savings that the buyers hope to make on the price they pay, then we are back to dealing with an equivalent model where instead of $V$ we use the random variable $R$. Note here that both $V$ and $h$ may be a random variable.

We may also have a situation where a bidder places a "time-out" on his offer so that the bid is withdrawn if the seller does not accept it before the time-out expires; however this case is not considered here, nor do we study cases where the seller varies the minimum sale price as a means to improve its income or accelerate the sale process. On the other hand, we study how the seller should choose this "decision time" so as to maximise his expected income over many successive sales, 
assuming that the value $V$ is a random variable. Thus we will examine the relation between the average value of this random "decision time" and the other parameters of this system assuming that similar sales take place repeatedly after a "rest period" that follows after each individual auction.

Another case which follows closely from the analysis in this section is when we consider that multiple units of the good are being sold at each auction. If the number of units sold at each successive auction are independent and identically distributed random variables, all the analysis we develop here follows through very directly to this case.

\subsection{The mathematical model}

Assume that after each bid, the seller waits for an exponentially distributed time of parameter $d$ to accept the bid. Since the exponential distribution has the memoryless property, with this assumption the potential buyers cannot use their ongoing observations of the seller to determine when it will decide to accept an offer, since the remaining decision time at any point in time has the same distribution as the initial decision time right after a bid is made. If a new bid arrives, then the process is repeated. Furthermore assume that after a bid is accepted, the auction has a random "rest time" of average value $R$, which is again modelled via an exponentially distributed time of rate $r=R^{-1}$.

The auction repeats itself an infinite number of times, and all random variables and processes that we discuss are assumed to be independent from each other in each of the successive auctions, although they will always have the same distribution. In particular, the value $V$ of the good beyond sold will be the random variable $V_{i}$ for the $i$-th successive auction, $i=1,2, \ldots$, with common distribution function $p(v)$.

Thus for any of the auctions in the sequence, we model this system as a continuous time Markov chain $\left\{X_{t}: t \geq 0\right\}$ with state space $\left\{0,1, \ldots, v, A_{1}, \ldots A_{V}\right\}$, where $V$ is itself a random variable (so that we are dealing with a doubly stochastic process).

Let $\left\{t_{1}, t_{2}, \ldots t_{n}, \ldots\right\}$ be the sequence of instants when the successive auctions begin. Then:

$-X_{t_{n}}=0$ corresponds to the value of the state when the $n-t h$ auction begins,

- The state $X_{t_{n}+t}=l$, for $t>0$ and $1 \leq l \leq v$, corresponds to the case at time $t_{n}+t$ during the $n-t h$ auction $\left(t_{n}+t<t_{n+1}\right)$ where $l$ bids have been received so that the offered price has reached the value $l$, while

$-X_{t}=A_{l}, t>0$, is the state at time $t_{n}+t$ during the $n-t h$ auction $\left(t_{n}+t<t_{n+1}\right)$ where the bid has been accepted at the price $l$.

Clearly, the transition rate from state $l$ to $l+1,1 \leq l \leq v-1$, is $\lambda$. The transition rate from state $l$ for $1 \leq l \leq v$ to state $A_{l}$ is $d$, and the transition rate from any state $A_{l}$ to state 0 is $r$.

In this model we assume that the good will be sold in each successive auction, and the main question is then to evaluate the income per unit time that the auctions generate for the seller.

$P_{v}($.$) denotes the stationary probability distribution of the Markov chain with$ state space $\left\{0,1, \ldots, v, A_{1}, \ldots, A_{v}\right\}$, where we assume that $V$ has a fixed value $v$. We will call this the reduced Markov chain, and denote by $\pi(l, v)$ the probability that the price that is obtained is $l$ given that the value of the good is $v$. 
The average rest time after the bid is accepted is the time spent in any of the states $A_{l}, 1 \leq l \leq v$; it is identical (and given by $r^{-1}$ ) for each auction. Thus $\pi(l, v)$ is the probability that the reduced Markov chain is in state $A_{l}$ given that it is in one of the states $\left\{A_{1}, \ldots, A_{v}\right\}$, hence:

$$
\pi(l, v)=\frac{P_{v}\left(A_{l}\right)}{\sum_{i=1}^{v} P_{v}\left(A_{i}\right)}, 1 \leq l \leq v .
$$

Assuming that the value of the good is a constant $v$, we can write the equations satisfied by the stationary probabilities $P_{v}($.$) as follows:$

$$
\begin{aligned}
P_{v}(l)(\lambda+d) & =\lambda P_{v}(l-1), 1 \leq l \leq v-1, \\
P_{v}(0) \lambda & =r \sum_{l=1}^{v} P_{v}\left(A_{l}\right), \\
P_{v}(v) d & =\lambda P_{v}(v-1), \\
P_{v}\left(A_{l}\right) r & =d P_{v}(l), \text { for } 1 \leq l \leq v, \\
1 & =P_{v}(0)+\sum_{l=1}^{v}\left[P_{v}(l)+P_{v}\left(A_{l}\right)\right] .
\end{aligned}
$$

If we write $\rho=\frac{\lambda}{\lambda+d}$, after some calculations these equations yield:

$$
\begin{aligned}
P_{v}(l) & =\rho^{l} P_{v}(0), 1 \leq l \leq v-1, \\
P_{v}(v) & =\frac{\lambda}{d} \rho^{v-1} P_{v}(0), \\
P_{v}\left(A_{l}\right) r & =d P_{v}(l), 1 \leq l \leq v, \\
P_{v}(0) & =\frac{r d}{r \lambda+\lambda d+r d} .
\end{aligned}
$$

As a consequence we can see that

$$
\sum_{l=1}^{v} P_{v}(l)=\frac{\lambda}{d} P_{v}(0),
$$

resulting in:

$$
\begin{aligned}
\pi(l, v) & =\frac{d}{\lambda} \rho^{l}, 1 \leq l \leq v-1 \\
\pi(v, v) & =\rho^{v-1}
\end{aligned}
$$

\subsection{Expected income}

Using the probabilities $\pi(l, v)$ we can compute the expected income from a single auction for a good whose value is $v$ directly as:

$$
\begin{aligned}
I_{v} & =\sum_{l=1}^{v} l \pi(l, v) \\
& =v \rho^{v-1}+\frac{1-v \rho^{v-1}+(v-1) \rho^{v}}{1-\rho}, \\
& =\frac{1-\rho^{v}}{1-\rho} .
\end{aligned}
$$

ACM Journal Name, Vol. 2, No. 3, 112006. 
so that the expected price brought by the auction is

$$
I=\frac{1-E\left[\rho^{V}\right]}{1-\rho}
$$

Comment 1 The formula (9) is very convenient because it says that average price of the good is formed by the probability distribution $V$ of the value that the bidders place on the good (and not just on the average of the value), and also by the bidding and selling rates.

Note that the total average time $A$ that the auction lasts is the average time it takes for a bid to be accepted, plus the rest time of average value $r^{-1}$, and is simply the total average time between two successive entries into state 0 . Since the average time spent in state 0 in each auction is $\lambda^{-1}$, we use (5) to obtain:

$$
\begin{aligned}
P_{v}(0) & =\frac{\lambda^{-1}}{A}, \\
A & =\frac{1}{P_{v}(0) \lambda}, \\
& =\frac{1}{\lambda}+\frac{r+d}{r d},
\end{aligned}
$$

which comes out to be independent of $v$ the value of the good. The average income per unit time brought by this auction is then $\Phi_{v}=I_{v} / A$ or

$$
\Phi_{v}=\frac{\lambda r(\lambda+d)}{\lambda r+\lambda d+r d}\left(1-\rho^{v}\right) .
$$

A simple reality check on the results obtained can be conducted as follows:

- Consider equation (13), and suppose that $v=+\infty$ so that the buyers do not have an upper bound on the price they are willing to offer; as a result the auction will be stopped by the seller's own decision to accept a bid. Furthermore, suppose that the seller is very anxious to make money so that there is no rest period, i.e. $r \rightarrow+\infty$. In that case we can easily see that the income per unit time is simply $\lambda$ or, in some sense, the rate at which the bidders add money to the offer.

-From (9) with $v=+\infty$ we see that the price that is obtained at an auction is

$$
I_{+\infty}=1+\frac{\lambda}{d}
$$

which makes sense because the price will be at least one, to which we should add the effect of any additional bids that may occur during the decision time. Thus the seller will always receive a price which is less than $1+\lambda / d$.

We can also readily obtain the average income per unit time $\Phi$ for the auction as a whole, under the assumption that the value of the good being sold is a random variable $V$ with probability distribution $\{p(v)\}$. We then have:

$$
\Phi=\frac{\lambda r(\lambda+d)}{\lambda r+\lambda d+r d}\left[1-E\left[\rho^{V}\right]\right],
$$

where $E\left[x^{V}\right]=\sum_{v=0}^{\infty} p(v) x^{v}$ is the generating function associated with the probability distribution $\{p(v)\}$. 
Comment 2 When we compare the formulas (9) and (15) we clearly see that if we use the decision rate $d$ to maximise income or income-per-unit time will lead to different results. However if the auction is "very busy" selling all the time, i.e. $r \rightarrow \infty$, we see that their ratio only depends on $\lambda$ and $d$ so that the best choice of $d$ will be the same for this case:

$$
\lim _{r \rightarrow \infty}\left[\frac{I}{\Phi}\right]=\frac{\lambda+d}{\lambda d}
$$

Of course, in the case $r \rightarrow \infty$ there is no rest period, and the seller is always busy running an auction.

\subsection{A numerical example}

It is interesting to observe how $\Phi$ varies as a function of $d$, as shown in the numerical examples of Figure 1. We select the value $V$ of the good to be uniformly distributed between 80 and 120 , we set $r=1$ to normalise the remaining parameters, and plot the income per unit time against the decision rate $d$ for different values of the arrival rate of the bids $\lambda$.

We see clearly that there is indeed an "optimum" value of $d$ which differs for each set of parameters and which maximises the income from the auction. Thus the seller can adjust his/her "patience" so that the rate of income is maximised. We also see that faster decisions (greater $d$ ) need to be made when the rate at which bids are made is higher. The curves show that there is some optimum value

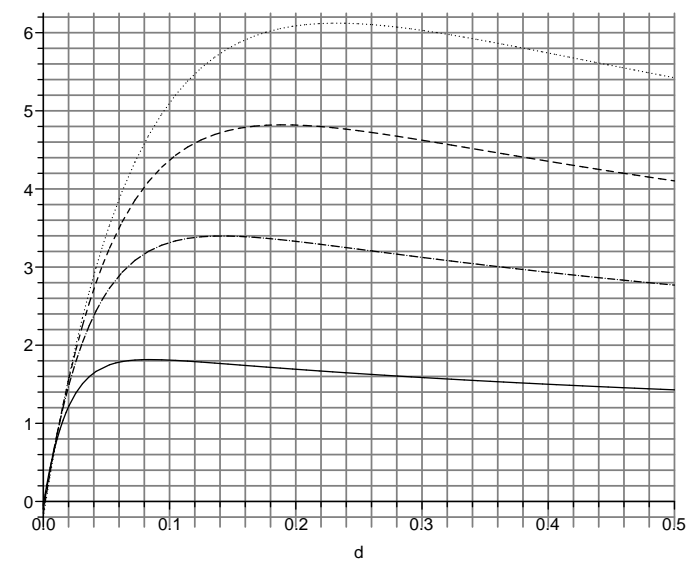

Fig. 1. Income per unit time versus the rate $\mathrm{d}$ at which decisions are made, for different values of the rate at which bids arrive ranging from 8 (top) to 2 (bottom). The value is uniformly distributed between 80 and 100. If the decision time is large ( $\mathrm{d}$ less than 0.1 ), the income per unit time can drop significantly.

of $d$ that will maximise the seller's income. Furthermore, when $\lambda$ is large we can afford to be less careful about waiting for some best value of the average decision time, and we can make decisions quite quickly ( $d$ large). 


\subsection{Increments of arbitrary size}

In the previous analysis we "make believe" that prices take integer values and bid increments are of unit size. This simplified model leads to more general results quite directly. Suppose that the successive values of the bids in an auction are denoted by $B_{i}>0, i=1,2 \ldots$ The increments are now $X_{1}=B_{1}>0$ and $X_{i+1}=B_{i+1}-B_{i}$ for $i \geq 1$. The random variable $V$ that we have previously used can now be interpreted as the number of bids received during an auction, with probability distribution $P(V=v)=p(v)$. If the $B_{i}$ are random variables too, then $B_{V}$ is a random variable representing the income that the auction will bring. Let $\bar{B}_{i}$ be the expected (average) value of the $i-t h$ bid. We first note that the expected income from the auction now is:

$$
I=\sum_{v=0}^{\infty} p(v) \sum_{l=1}^{v} \bar{B}_{l} \pi(l, v)
$$

If the increments have a common average value $\bar{X}$ then $\bar{B}_{l}=l \bar{X}$, we have:

$$
I=\sum_{v=0}^{\infty} p(v) \sum_{l=1}^{v} l \bar{X} \pi(l, v) .
$$

and the analysis reduces to the earlier case so that using (9) we obtain:

$$
\begin{aligned}
I & =\bar{X} \frac{1-\rho^{v}}{1-\rho}, \\
\Phi & =\bar{X} \frac{\lambda r(\lambda+d)}{\lambda r+\lambda d+r d}\left[1-E\left[\rho^{V}\right]\right] .
\end{aligned}
$$

\subsection{The rate at which bids arrive}

It is also of interest to study the effect of "repeated bids" which include bids being made from previously unsuccesful bidders as well as from new bidders. Thus for a given auction, bids arrive at a total rate $\lambda$ and this differs from the rate $\gamma$, at which bids are made for the first time by individual bidders, because a given bidder who is outbid by someone else may try again. In this section we discuss how $\gamma$ and $\lambda$ are related. Let $\alpha$ be the stationary probability that a given auction is active, and $(1-\alpha)$ be the probability that it is at rest before the next auction begins. During the rest period, we assume that bids are not made to this particular auction since bidders are aware that the auction is not open.

We know that out of $\Phi$ bids per unit time, only one will be successful and therefore the corresponding bidder will not return to the auction since he has been able to buy the good. Thus the probability that a bid is unsuccessful is $(\Phi-1) / \Phi$. Thus when the auction is open, the rate at which bids arrive is:

$$
\begin{aligned}
\lambda & =\gamma+\lambda p \frac{\Phi-1}{\Phi}, \\
& =\gamma \frac{1}{(1-p)+\frac{p}{\Phi}} .
\end{aligned}
$$

where $p$ is the probability that an unsuccessful bidder will try again with a new (and higher) bid. Thus the rate at which bids are made will in general be much 
larger than the rate at which bidders arrive to the auction: $\lambda>>\gamma$. Furthermore, equations (22) and (15) tell us that the actual rate of bids depends on the number of bids that are made, and vice-versa. This analysis is illustrated in Figure 2 where we see that for low values of $\gamma$ the income per unit time from the auction is just not "taking off", and when some threshold is exceeded it attains much higher values. It can also be seen that when $\gamma$ is large, $\lambda$ will grow linearly as a function of $\gamma$

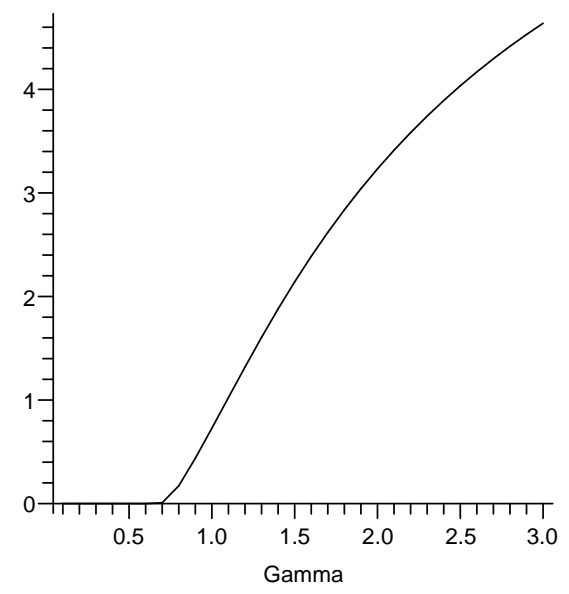

Fig. 2. Income per unit time (Phi) versus the rate Gamma at which bidders arrive. Here $\mathrm{d}=0.1$, $\mathrm{p}=0.7$ and $\mathrm{r}=1$.

because $\Phi$ will be larger and will therefore have less impact on the value of $\lambda$ as shown in expression (22).

\subsection{State dependent arrival rate}

Assume now that the arrival rate of bids $\lambda_{i}$ depends on the price that the most recent bid has reached, which is an example of the bidders' rationality, and similarly let the decision rate $d_{i}$ depend on the price that is being considered by the seller which is again an example of rational choice. The analysis only requires a minor modification in the previously studied model when the value of the good is a constant $v$ :

$$
\begin{aligned}
P_{v}(l)\left(\lambda_{l}+d_{l}\right) & =\lambda_{l-1} P_{v}(l-1), 1 \leq l \leq v-1, \\
P_{v}(0) \lambda_{0} & =r \sum_{l=1}^{v} P_{v}\left(A_{l}\right), \\
P_{v}(v) d_{v} & =\lambda_{v-1} P_{v}(v-1), \\
P_{v}\left(A_{l}\right) r & =d_{l} P_{v}(l), \text { for } 1 \leq l \leq v \\
1 & =P_{v}(0)+\sum_{l=1}^{v}\left[P_{v}(l)+P_{v}\left(A_{l}\right)\right] .
\end{aligned}
$$


If we write $\rho_{i}=\frac{\lambda_{i-1}}{\lambda_{i}+d_{i}}$, we obtain:

$$
\begin{aligned}
P_{v}(l) & =P_{v}(0) \Pi_{i=1}^{l} \rho_{i}, 1 \leq l \leq v-1, \\
P_{v}(v) & =P_{v}(0) \frac{\lambda_{v-1}}{d_{v}} \Pi_{i=1}^{v-1} \rho_{i}, \\
P_{v}\left(A_{l}\right) r & =d_{l} P_{v}(l), \text { for } 1 \leq l \leq v .
\end{aligned}
$$

Comment 3 Note that the state dependent model also reflects some form of rational behaviour on the part of bidders who decide to bid at the auction depending on what the price of the most recent bid is. Thus one would expect that the bid rate might in some cases drop as the price increases, or yet in others we may discover and increase of the rate and then a decrease, since buyers often are more attracted by goods whose price may relate to the value they associate with the good. Thus we may imagine that $\lambda_{i+1}>\lambda_{i}$ if $\operatorname{Prob}[i>V]$ is larger than $0 . f$, while the opposite would happen if $\operatorname{Prob}[i \leq V]$ is higher.

\section{VARIATIONS ON THE SINGLE AUCTION MODEL}

Many other variations and generalisations of the basic models that were discussed earlier can be considered. For instance, one case that we have not considered is when each successive bid is required to exceed a certain minimum value. We will not detail this case in this paper.

Another interesting case we now analyse is the "Vickrey" auction where the highest bidder is selected but the good is sold at the second highest price price [McAfee and McMillan 1987], except for the first bid which (if accepted) will be accepted at its offered value. By applying the previous approach to a Vickrey auction, the expected income $I_{v}^{\nu}$ it will bring for a good of value $v$ becomes:

$$
\begin{aligned}
I_{v}^{\nu} & =\sum_{l=2}^{v}(l-1) \pi(l, v)+\pi(1, v), \\
& =\frac{1-\rho^{v}}{1-\rho}-1+\frac{d}{\lambda} \rho, \\
& =\rho\left[\frac{1-\rho^{v-1}}{1-\rho}+\frac{d}{\lambda}\right],
\end{aligned}
$$

The average income per unit time is then $\Phi_{v}^{\nu}=I_{v}^{\nu} / A$, leading to the average income per unit time $\Phi^{\nu}$ for the Vickrey auction when the value of the good being sold is a random variable $V$ with probability distribution $\{p(v)\}$, and using the fact that the average duration of each auction is the same as in the "English" case, we have:

$$
\Phi^{\nu}=\frac{\lambda r d}{r d+\lambda(r+d)}\left[(1-\rho)+\frac{\lambda}{d}-\left(1+\frac{\lambda}{d}\right) E\left[\rho^{V}\right]\right] .
$$

Numerical examples that compare the ordinary English auction with a Vickrey auction are shown below, indicating that the optimum incomes per unit time are quite close in both cases. 


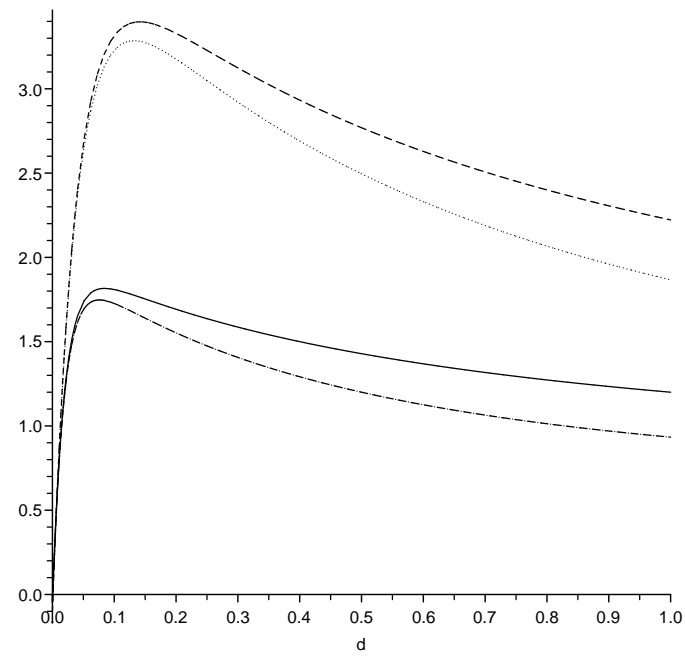

Fig. 3. Income per unit time versus the rate $d$ at which decisions are made for the English and Vickrey auction with unit increments. The value of the good is uniformly distributed between 80 and 100, and the arrival rate of bids arrive is 4 (top) and 2 (bottom).

\subsection{An English auction with a seller reservation price}

Most auctions will start with a seller's reservation price, call it $s$. If no bid is made for at least that price by the seller's decision time, then the auction ends without a sale. This case was not considered in the previous analysis in which the seller may wait indefinitely for the initial bid with $s=1$.

Here we will denote by $P_{v}\left(A_{0}\right)$ the probability that the seller stops the auction because a bid with a price of at least $s$ has not been received. When this happens, the auction "rests" for some time of average value $r^{-1}$ and then restarts. In this case it is also normal to consider that the rate at which bids are made is a function of this threshold price, call it $\lambda_{s}$. Of course, unless the value $v$ that buyers associate with the good is at least $s$, this auction will never result in a sale. Therefore we will take $v \geq s$.

The equations satisfied by the state probabilities now become:

$$
\begin{aligned}
P_{v}(l)\left(\lambda_{s}+d\right) & =\lambda_{s} P_{v}(l-1), s<l \leq v-1 \\
P_{v}(s)\left(\lambda_{s}+d\right) & =\lambda_{s} P_{v}(0), \\
P_{v}(0)\left(\lambda_{s}+d\right) & =r \sum_{l=s}^{v} P_{v}\left(A_{l}\right)+r P_{v}\left(A_{0}\right), \\
P_{v}(v) d & =\lambda_{s} P_{v}(v-1), \\
P_{v}\left(A_{l}\right) & =\frac{d}{r} P_{v}(l), s \leq l \leq v, l=0, \\
P_{v}(0)+P_{v}\left(A_{0}\right) & +\sum_{l=s}^{v}\left[P_{v}(l)+P_{v}\left(A_{l}\right)\right]=1 .
\end{aligned}
$$


If we set $\rho=\lambda_{s} /\left(\lambda_{s}+d\right)$ we obtain:

$$
\begin{aligned}
P_{v}(l) & =\rho^{l-s+1} P_{v}(0), s \leq l \leq v-1, \\
P_{v}(v) & =\frac{\lambda_{s}}{d} \rho^{v-s} P_{v}(0), \\
P_{v}\left(A_{l}\right) & =\frac{d}{r} P_{v}(l), s \leq l \leq v, \text { or } l=0, \\
P_{v}(0) & =\frac{r d}{\left(\lambda_{s}+d\right)(r+d)} .
\end{aligned}
$$

We are also interested in the probability that the price obtained is $l$ given that the value of the good is $v$, with $v \geq s$. In that case we will have:

$$
\begin{gathered}
\pi(l, v)=\frac{P_{v}\left(A_{l}\right)}{P_{v}\left(A_{0}\right)+\sum_{i=s}^{v} P_{v}\left(A_{l}\right)}, s \leq l \leq v, l=0, \\
\pi(l, v)=\frac{d}{d+\lambda_{s}} \rho^{l-s+1}, s \leq l \leq v-1, \\
=\rho^{v-s+1}, l=v, \\
=\frac{d}{d+\lambda_{s}}, l=0,
\end{gathered}
$$

so that the income from a single auction for a good whose value is $v \leq s$ is now:

$$
I_{v}=\rho\left[s+\frac{\lambda_{s}}{d}\left(1-\rho^{v-s}\right)\right]
$$

The numerical results in Figure 4 show that having a seller reservation price can

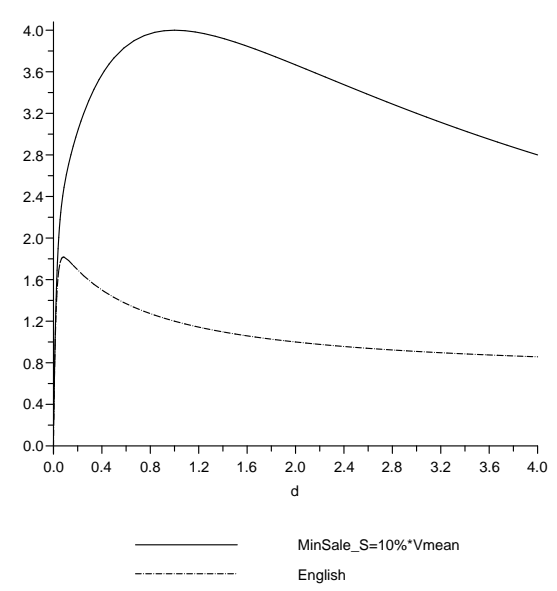

Fig. 4. Income per unit time versus the rate $\mathrm{d}$ at which decisions are made for an auction without a seller reservation price (lower curve), and for one where it is 10 (curve above). In both cases bids arrive at rate $\lambda=2$. The value $\mathrm{V}$ is uniformly distributed between 80 and 100 . The seller reservation price has the effect of greatly improving the income per unit time, even though some of the auctions may end without a sale. 
yield much higher income per unit time, provided that decisions are taken faster (larger d) than in an auction without it.

\subsection{A deterministic model and heuristic rule}

Consider an auction that begins at time $t=0$ with successive bids arriving at $0 \leq a_{1} \leq a_{2} \leq \ldots$. Let $\alpha_{n+1}=a_{n+1}-a_{n}$. The $X_{n}>0, n \geq 1$ are the increments that successive bids offer so that the value of the $n-t h$ bid is $B_{n}=\sum_{i=1}^{n} X_{i}$. Suppose the seller decides to accept the $n$th bid at time $a_{n}+D_{n}$ provided no new bid arrives by that time. If $W_{n}$ is the total wait time of the $n$th bidder, we see that:

$$
\begin{aligned}
W_{n} & =\alpha_{n+1}, \text { if } D_{n}>\alpha_{n+1}, \\
& =D_{n}, \text { otherwise. }
\end{aligned}
$$

since a bidder may leave the bidding process, if he so wishes, as soon as a higher bid is made.

We can also study how a decision may be made by the seller who is observing the successive bids. We may expect that the $n$th increment $X_{n}$ will depend on the previous bid $B_{n-1}$, so that as time goes by at some point the bidding price has reached a high enough value, but the relative increase in the bid has somehow reached a point of diminishing returns. If the seller is interested in the income per unit time, he may well decide to accept the $k+1$ th offer if:

$$
\begin{aligned}
\frac{B_{k}}{a_{k}} & >\frac{B_{k-1}}{a_{k-1}}, \text { and } \\
\frac{B_{k+1}}{a_{k+1}} & <\frac{B_{k}}{a_{k}} .
\end{aligned}
$$

Equivalently, he accepts the $z$ th bid where:

$$
z=\inf \left\{n: \frac{X_{n}}{B_{n-1}}<\frac{\alpha_{n}}{a_{n-1}}\right\},
$$

when the relative increase in the value of the bid is less than the relative increase in the time that the seller waits for the bid.

\section{NETWORKED AUCTIONS}

We complete this "tour d'horizon" of new probabilistic auction models by showing how these models can be extended to the case where many English auctions are being conducted by different sellers, and bidders can move different auctions to try to make a purchase. Although we are imagining that all these auctions are selling the same type of good, the analysis would be similar if the networked system would include auctions for different types of goods. Then consider $N$ auctions which are running concurrently, and let

$$
n(t)=\left(n_{1}(t), \ldots, n_{N}(t)\right)
$$

be the vector representing the number of potential bidders $n_{i}(t)$ at the $i$ th auction at time $t \geq 0$, while the vector

$$
k(t)=\left(k_{1}(t), . ., k_{N}(t)\right)
$$


denotes the price currently attained by the most recent bids at the $N$ auctions, and $k_{i}(t)$ is the price that the $i$ th auctions have reached at time $t$, so that the state of the system as a whole is the pair of vectors $(n(t), k(t))$, which will have the following constraints:

- If $k_{i}(t)>0$, this means that there is at least one current bid at auction $i$; as a consequence we must have $n_{i}(t)>0$ as well since there must be at least one potential customer present.

-If $n_{i}(t)=0$ then $k_{i}(t)=0$, while if $k_{i}(t)=0$ then we may have any value $n_{i}(t) \geq 0$.

- If $n_{i}(t)>0$ it is possible that no bid has yet been received at auction $i$ or that the most recent bid has been accepted and the successful bidder has returned to the set of other bids, so that we may have $k_{i}(t)=0$.

We introduce the Mobile Bidder Model (MBM) where any bidder, except for the one who has made the most recent bid and is waiting for a response from the seller, is allowed to move at any time from one auction to another one. Of course, all other bidders are also allowed to make a bid. Bidders who leave one auction can either go to another one or they can depart from the auction system as a whole. In this model, the mobility of bidders will be represented by a Markov chain with transition probabilities $P(i, j)$ where $i \in\{1, \ldots N\}$ and $j \in\{1, \ldots N, D\}$, where $D$ denotes the departure point of a bidder from the system, so that

$$
\sum_{j=1}^{N} P(i, j)+P(i, D)=1,
$$

for any $i=1, \ldots N$. Furthermore, bidders join auction $i$ from the "outside world" according to a Poisson process.

The MBM is represented as follows, where we denote by $y$ the number of bidders and by $x$ the level attained by the bid:

-Bidders arrive from the "outside world" to the $i$ th auction according to a Poisson process of rate $\gamma_{i}$.

- The rate of departure from auction $i$ of a bidder, except for the one that has made the current highest bid, is $\mu_{i} \geq 0$ so that with probability $\mu_{i} \Delta t+o(\Delta t)$ any one bidder leaves the auction in the time interval $[t, t+\Delta t[$. If there is currently no outsanding bid, all bidders can depart. Thus the rate of departure is a function of $(y, x)$ as follows:

$$
\begin{aligned}
& \mu_{i}(y, x)=(y-1) \mu_{i}, x>0 \\
& \mu_{i}(y, 0)=y \mu_{i} .
\end{aligned}
$$

A bidder departing from auction $i$ will head to auction $j$, or to the outside of the network if $j=D$, with probability $P(i, j)$, where $j \in\{1, \ldots N, D\}$.

- The value of the good being sold at auction $i$ is the random variable $V_{i}$ which is independent of other quantities in the system, and its probability distribution is $p_{i}(v)=\operatorname{Prob}\left[V_{i}=v\right]$, and let $\psi_{i, x}=\operatorname{Prob}\left[x<V_{i}\right]$ with $\psi_{i, 0}=1$. New bids will stop being made when the most recent bid has attained the value of the good. 
Thus the rate at which bidders at auction $i$ make bids is a function of $(\mathrm{y}, \mathrm{x})$ as follows:

$$
\begin{aligned}
& \beta_{i}(y, x)=(y-1) \beta_{i} \psi_{i, x} x>0, \\
& \beta_{i}(y, 0)=\beta_{i} y .
\end{aligned}
$$

where $\beta_{i}>0$. Again for any individual bidder we assume exponentially distributed bidding times, independent of others' bids and of the same bidder's previous bids.

- Finally, the rate at which a bid is accepted at auction $i$ is $\delta_{i}$, and the bid acceptance times are independent and identically distributed random variables. When a bid is accepted at auction $i$ the successful bidder joins the set of bidders at the same auction, and auction $i$ starts again with all the bidders. Notice that the most recently winning bidder's behaviour henceforth is the same as that of the other bidders.

Comment 4 The MBM includes several aspects which represent choices made by the potential bidders. These include $\gamma_{i}$ which is the rate at which bidders first choose to visit the $i$ th auction, $V_{i}$ the random variable representing the value of the good at auction $i$ as perceived by the bidders, $\beta_{i}$ the rate at which bidders at auctioon $i$ make bids, $\mu_{i}$ the rate at which a potential bidder at auction $i$ chooses to leave that auction, and finally $P(i, j)$ the probability that a bidder leaving auction $i$ then chooses to go to auction $j$. All of these quantities can represent rational choices based on the trust that a bidder can place on the different auctions, or on the quality of the goods that are being sold. However at this point we do not assume that these quantities depend on the instantaneous state of all of the auctions, e.g. the price currently attained and the number of bidders that are present at each of the auctions. These additional aspects of rational choice will be discussed in Section 4.2 .

\subsection{Deriving the system equations}

With these assumptions, $\{(n(t), k(t)), t \geq 0\}$ is a continuous time, discrete statespace Markov chain [Medhi 1994]. We denote its probability distribution by $p(n, k, t)=$ $\operatorname{Prob}[n(t)=n, k(t)=k]$ for some given initial condition at $t=0$.

Let us introduce the following notation. $e_{i}$ will denote the $n$-vector all of whose elements are zero, except for the $i$ th element which is +1 , and let $n_{i}^{+}=n+e_{i}$, $n_{i}^{-}=n-e_{i}$ provided that $n_{i}>0, n_{i j}^{+-}=n+e_{i}-e_{j}$ provided that $n_{j}>0$. Similarly $k_{i}^{+}=k+e_{i}$ and $k_{i}^{-}=k-e_{i}$ provided that $k_{i}>0$.

The Chapman-Kolmogorov $(\mathrm{C}-\mathrm{K})$ equations for the system are:

$$
\begin{aligned}
& \frac{d p(n, k, t)}{d t}=\sum_{i=1}^{N}\left\{\gamma_{i} p\left(n_{i}^{-}, k, t\right) 1\left[n_{i}>0\right]\right. \\
+ & \beta_{i}\left(n_{i}-1\right) \psi_{i}\left(k_{i}-1\right) p\left(n, k_{i}^{-}, t\right) 1\left[n_{i}>0, k_{i} \geq 2\right] \\
+ & \beta_{i} \psi_{i, 0} n_{i} p\left(n, k_{i}^{-}, t\right) 1\left[k_{i}=1\right] \\
+ & \mu_{i} P(i, D) p\left(n_{i}^{+}, k, t\right)\left(n_{i} 1\left[k_{i}>0\right]+\left(n_{i}+1\right) 1\left[k_{i}=0\right]\right)
\end{aligned}
$$




$$
\begin{aligned}
& +\sum_{j=1}^{N} \mu_{i} n_{i} P(i, j) p\left(n_{i j}^{+-}, k, t\right) 1\left[n_{j}>0, k_{i}>0\right] \\
& +\sum_{j=1}^{N} \mu_{i}\left(n_{i}+1\right) P(i, j) p\left(n_{i j}^{+-}, k, t\right) 1\left[k_{i}=0\right] \\
& \left.+\sum_{x=1}^{\infty} \delta_{i} p\left(n, k+x e_{i}\right) 1\left[k_{i}=0\right] 1\left[n_{i}>0\right]\right\} \\
& -\sum_{i=1}^{N}\left\{\gamma_{i}+\left[\left(\delta_{i}+\mu_{i}\left(n_{i}-1\right)\right) 1\left[n_{i}>0, k_{i}>0\right]\right.\right. \\
& \left.\quad+\beta_{i} \psi_{i}\left(k_{i}\right)\left(n_{i}-1\right)\right] 1\left[n_{i}>0, k_{i}>0\right] \\
& \left.\quad+\left(\beta_{i} n_{i}+\mu_{i} n_{i}\right)\left[n_{i}>0, k_{i}=0\right]\right\} p(n, k, t)
\end{aligned}
$$

Note that the term beginning with $\delta_{i}$ in the 7 -th line of the equation corresponds to the transitions which occur when bids are accepted, while the last three lines correspond to the rates of the events that do not take place. Now define:

$$
\begin{aligned}
p(n, t) & =\sum_{i=1}^{N} \sum_{k_{i} \geq 0} p(n, k, t), \\
p\left(n, k_{i}=0, t\right) & =\sum_{j=1, j \neq i}^{N} \sum_{k_{j} \geq 0} p(n, k, t) 1\left[k_{i}=0\right],
\end{aligned}
$$

and sum the equation (51) over all vectors $k$ to obtain:

$$
\begin{aligned}
& \frac{d p(n, t)}{d t}=\sum_{i=1}^{N}\left\{\gamma_{i} p\left(n_{i}^{-}, t\right) 1\left[n_{i}>0\right]\right. \\
+ & \mu_{i} n_{i} P(i, D) p\left(n_{i}^{+}, t\right) \\
+ & \mu_{i} P(i, D) p\left(n_{i}^{+}, k, t\right) 1\left[k_{i}=0\right] \\
+ & \sum_{k_{i}=1}^{\infty} \sum_{j=1, j \neq i}^{N} \sum_{k_{j}=0}^{\infty} \beta_{i}\left(n_{i}-1\right) \psi_{i}\left(k_{i}\right) p(n, k, t) 1\left[n_{i}>0\right] \\
+ & \beta_{i} n_{i} p(n, k, t) 1\left[k_{i}=0\right] 1\left[n_{i}>0\right] \\
+ & \sum_{j=1}^{N}\left[\mu_{i} n_{i} P(i, j) p\left(n_{i j}^{+-}, t\right) 1\left[n_{j}>0\right]\right. \\
+ & \left.\mu_{i} P(i, j) p\left(n_{i j}^{+-}, k, t\right) 1\left[k_{i}=0\right] 1\left[n_{j}>0\right]\right] \\
+ & \left.\delta_{i}\left[p(n, t)-p(n, k, t) 1\left[k_{i}=0\right]\right] 1\left[n_{i}>0\right]\right\} \\
- & \sum_{i=1}^{N}\left\{\gamma_{i} p(n, t)\right. \\
+ & \delta_{i}\left[p(n, t)-p(n, k, t) 1\left[k_{i}=0\right]\right] 1\left[n_{i}>0\right] \\
+ & \mu_{i}\left(n_{i}-1\right)\left[p(n, t)-p(n, k, t) 1\left[k_{i}=0\right]\right] 1\left[n_{i}>0\right]
\end{aligned}
$$




$$
\begin{aligned}
& +\sum_{k_{i}=1}^{\infty} \sum_{j=1, j \neq i}^{N} \sum_{k_{j}=0}^{\infty} \beta_{i} \psi\left(k_{i}\right)\left(n_{i}-1\right) p(n, k, t) 1\left[n_{i}>0\right] \\
& \left.+\left(\mu_{i}+\beta_{i}\right) n_{i} p(n, k, t) 1\left[k_{i}=0\right]\right\} \\
& =\sum_{i=1}^{N}\left\{\gamma_{i} p\left(n_{i}^{-}, t\right) 1\left[n_{i}>0\right]\right. \\
& +\mu_{i} n_{i} P(i, D) p\left(n_{i}^{+}, t\right) \\
& +\mu_{i} P(i, D) p\left(n_{i}^{+}, k, t\right) 1\left[k_{i}=0\right] \\
& +\sum_{j=1}^{N}\left[\mu_{i} n_{i} P(i, j) p\left(n_{i j}^{+-}, t\right) 1\left[n_{j}>0\right]\right. \\
& \left.\left.+\mu_{i} P(i, j) p\left(n_{i j}^{+-}, k\right) 1\left[k_{i}=0\right] 1\left[n_{j}>0\right]\right]\right\} \\
& -\sum_{i=1}^{N}\left\{\left(\gamma_{i}+\mu_{i}\left(n_{i}-1\right) 1\left[n_{i}>0\right]\right) p(n, t)\right\}
\end{aligned}
$$

After grouping terms which correspond to states with $k_{i}=0$ we obtain:

$$
\begin{aligned}
& \frac{d p(n, t)}{d t}=\sum_{i=1}^{N}\left\{\gamma_{i} p\left(n_{i}^{-}, t\right) 1\left[n_{i}>0\right]\right. \\
+ & \mu_{i} n_{i} P(i, D) p\left(n_{i}^{+}, t\right) \\
+ & \sum_{j=1}^{N}\left[\mu_{i} n_{i} P(i, j) p\left(n_{i j}^{+-}, t\right) 1\left[n_{j}>0\right]\right. \\
+ & \sum_{i=1}^{N}\left\{-\left(\gamma_{i}+\mu_{i}\left(n_{i}-1\right) 1\left[n_{i}>0\right]\right) p(n, t)\right. \\
+ & \mu_{i} P(i, D) p\left(n_{i}^{+}, k, t\right) 1\left[k_{i}=0\right] \\
+ & \sum_{j=1}^{N} \mu_{i} n_{i} P(i, j) p\left(n_{i j}^{+-}, k_{i}=0, t\right) 1\left[n_{j}>0\right] \\
- & \left.\mu_{i} p\left(n, k_{i}=0, t\right) 1\left[n_{i}>0\right]\right\}
\end{aligned}
$$

\subsection{Approximate stationary solution of the MBM when bidders are very active}

Under the Active Bidders Assumption (ABA), we suppose that that

$$
p(n, k, t) 1\left[k_{i}=0\right]<<p(n, t) \quad \text { if } n_{i}>0,
$$

i.e., for any $(n, k)$ such that $n_{i}>0$ and $k_{i}=0$. We are thus assuming that the probability that there are no bids when there is at least one bidder at an auction is very small compared to the overall probability of the same state. The equations (57) then become:

$$
\begin{aligned}
\frac{d p(n, t)}{d t} & \approx \sum_{i=1}^{N}\left\{\gamma_{i} p\left(n_{i}^{-}, t\right) 1\left[n_{i}>0\right]\right. \\
& +\mu_{i} n_{i} P(i, D) p\left(n_{i}^{+}, t\right)
\end{aligned}
$$




$$
\begin{aligned}
& +\sum_{j=1}^{N}\left[\mu_{i} n_{i} P(i, j) p\left(n_{i j}^{+-}, t\right) 1\left[n_{j}>0\right]\right. \\
& \left.-\left[\gamma_{i}+\mu_{i}\left(n_{i}-1\right)\right] p(n, t) 1\left[n_{i}>0\right]\right\}
\end{aligned}
$$

Result 1 The stationary solution of equations (57) for the MBM under the ABA are obtained by setting $\frac{d p(n, t)}{d t}=0$ for all $n_{i}>0$ in (60) and is given by:

$$
p(n) \approx \prod_{i=1}^{N} \frac{e^{-U_{i}}}{U_{i}} \frac{U_{i}^{n_{i}}}{\left(n_{i}-1\right) !}
$$

where $U_{i}=\frac{\Lambda_{i}}{\mu_{i}}$ and the $\Lambda_{i}$ are the solution of the system of linear equations:

$$
\Lambda_{i}=\gamma_{i}+\sum_{j=1}^{N} \Lambda_{j} P(j, i), i=1, \ldots, N
$$

Furthermore, in steady state the average number of bidders at the $i$ th auction is given by $\left\langle n_{i}\right\rangle \approx\left[1+U_{i}\right]$.

Proof The proof is direct by substitution of (61) in (60) which yields:

$$
\begin{aligned}
0 \approx & \sum_{i=1}^{N}\left\{\gamma_{i} \mu_{i} \frac{n_{i}-1}{\Lambda_{i}}+\mu_{i} n_{i} P(i, D) \frac{\Lambda_{i}}{\mu_{i} n_{i}}\right. \\
& \left.+\sum_{j=1}^{N} \mu_{i} n_{i} P(i, j) \frac{\mu_{j}\left(n_{j}-1\right) \Lambda_{i}}{\Lambda_{j} \mu_{i} n_{i}}-\gamma_{i}-\mu_{i}\left(n_{i}-1\right)\right\} \\
\approx & \sum_{i=1}^{N}\left\{\gamma_{i} \mu_{i} \frac{n_{i}-1}{\Lambda_{i}}+P(i, D) \Lambda_{i}+\sum_{j=1}^{N} P(i, j) \frac{\mu_{j}\left(n_{j}-1\right) \Lambda_{i}}{\Lambda_{j}}-\gamma_{i}-\mu_{i}\left(n_{i}-1\right)\right\}
\end{aligned}
$$

Using (62), and with $P(i, D)=1-\sum_{j=1}^{N} P(i, j)$ so that

$$
\sum_{i=1}^{N} \Lambda_{i} P(i, D)=\sum_{i=1}^{N} \gamma_{i}
$$

we write

$$
0 \approx \sum_{i=1}^{N}\left\{\gamma_{i} \mu_{i} \frac{n_{i}-1}{\Lambda_{i}}+\sum_{j=1}^{N} \frac{\mu_{j}\left(n_{j}-1\right)\left(\lambda_{j}-\gamma_{j}\right)}{\lambda_{j}}-\mu_{i}\left(n_{i}-1\right)\right\}
$$

where the right-hand-side is zero. Finally note that $e^{-U_{i}} / U_{i}$ is the normalising constant that guarantees that the sum of the probabilities equals one. Thus $<$ $n_{i}>\approx\left[1+U_{i}\right]$ is obtained from

$$
<n_{i}>\approx \frac{e^{-U_{i}}}{U_{i}} \sum_{x=1}^{\infty} x \frac{U_{i}^{x}}{(x-1) !}
$$




\subsection{The solution when bids and sales are very frequent}

In this section we will consider the case when bidding and selling occurs more frequently than the bidders' moving between auction centres and into our out of the auction centre.Thus we will consider the case where for all $i=1, \ldots, N$,

$$
\Lambda_{i}, \mu_{i}<<\delta_{i}, \beta_{i} \psi_{i, k_{i}}
$$

which also implies that $\gamma_{i}<<\delta_{i}, \beta_{i} \psi_{i, k_{i}}$. Considering the solution for:

$$
p(n, k)=\lim _{t \rightarrow \infty} p(n, k, t),
$$

and starting with (51) expressed in steady-state:

$$
\begin{aligned}
& \sum_{i=1}^{N}\left\{\gamma_{i} p\left(n_{i}^{-}, k\right) 1\left[n_{i}>0\right]\right. \\
+ & \beta_{i}\left(n_{i}-1\right) \psi_{i}\left(k_{i}-1\right) p\left(n, k_{i}^{-}\right) 1\left[n_{i}>0, k_{i} \geq 2\right] \\
+ & \beta_{i} \psi_{i, 0} n_{i} p\left(n, k_{i}^{-}\right) 1\left[k_{i}=1\right] \\
+ & \mu_{i} P(i, D) p\left(n_{i}^{+}, k\right)\left(n_{i} 1\left[k_{i}>0\right]+\left(n_{i}+1\right) 1\left[k_{i}=0\right]\right) \\
+ & \sum_{j=1}^{N} \mu_{i} n_{i} P(i, j) p\left(n_{i j}^{+-}, k\right) 1\left[n_{j}>0, k_{i}>0\right] \\
+ & \sum_{j=1}^{N} \mu_{i}\left(n_{i}+1\right) P(i, j) p\left(n_{i j}^{+-}, k\right) 1\left[k_{i}=0\right] \\
+ & \left.\sum_{x=1}^{\infty} \delta_{i} p\left(n, k+x e_{i}\right) 1\left[k_{i}=0\right] 1\left[n_{i}>0\right]\right\} \\
= & \sum_{i=1}^{N}\left\{\gamma_{i}+\left[\left(\delta_{i}+\mu_{i}\left(n_{i}-1\right)\right) 1\left[n_{i}>0, k_{i}>0\right]\right.\right. \\
& \left.\quad+\beta_{i} \psi_{i, k_{i}}\left(n_{i}-1\right)\right] 1\left[n_{i}>0, k_{i}>0\right] \\
& \left.+\left(\beta_{i} n_{i}+\mu_{i} n_{i}\right) 1\left[n_{i}>0, k_{i}=0\right]\right\} p(n, k)
\end{aligned}
$$

we remove terms which are relatively small due to the condition (67) to obtain:

$$
\begin{aligned}
& \sum_{i=1}^{N}\left\{\beta_{i}\left(n_{i}-1\right) \psi_{i, k_{i}-1} p\left(n, k_{i}^{-}\right) 1\left[n_{i}>0, k_{i} \geq 2\right]\right. \\
&+\left.\beta_{i} \psi_{i, 0} n_{i} p\left(n, k_{i}^{-}\right) 1\left[k_{i}=1\right]+\sum_{x=1}^{\infty} \delta_{i} p\left(n, k+x e_{i}\right) 1\left[k_{i}=0\right] 1\left[n_{i}>0\right]\right\} \\
&= \sum_{i=1}^{N}\left\{\left[\left(\delta_{i}+\mu_{i}\left(n_{i}-1\right)\right) 1\left[n_{i}>0, k_{i}>0\right]\right.\right. \\
&\left.\quad+\beta_{i} \psi_{i, k_{i}}\left(n_{i}-1\right)\right] 1\left[n_{i}>0, k_{i}>0\right] \\
&\left.\quad+\beta_{i} n_{i} 1\left[n_{i}>0, k_{i}=0\right]\right\} p(n, k)
\end{aligned}
$$

and then write $p(n, k)=p(k \mid n) p(n)$ and divide both sides by $p(n)$ to obtain:

$$
\sum_{i=1}^{N}\left\{\beta_{i}\left(n_{i}-1\right) \psi_{i, k_{i}-1} p\left(k_{i}^{-} \mid n\right) 1\left[n_{i}>0, k_{i} \geq 2\right]\right.
$$




$$
\begin{aligned}
& \left.+\beta_{i} \psi_{i, 0} n_{i} p\left(k_{i}^{-} \mid n\right) 1\left[k_{i}=1\right]+\sum_{x=1}^{\infty} \delta_{i} p\left(k+x e_{i} \mid n\right) 1\left[k_{i}=0\right] 1\left[n_{i}>0\right]\right\} \\
& \approx \sum_{i=1}^{N}\left\{\left[\left(\delta_{i}+\mu_{i}\left(n_{i}-1\right)\right) 1\left[n_{i}>0, k_{i}>0\right]\right.\right. \\
& \left.\left.\quad+\beta_{i} \psi_{i, k_{i}}\left(n_{i}-1\right)\right] 1\left[n_{i}>0, k_{i}>0\right]+\beta_{i} n_{i} 1\left[n_{i}>0, k_{i}=0\right]\right\} p(k \mid n)
\end{aligned}
$$

As an immediate consequence we have:

Result 2 The solution $p(k \mid n)$ for $n_{i}>0$ and $k_{i}>0, i=1, \ldots N$ to (73) is given by:

$$
\begin{aligned}
p(k \mid n) & \approx \prod_{i=1}^{N} p_{i}\left(k_{i} \mid n_{i}\right), \text { where } \\
p_{i}\left(k_{i} \mid n_{i}\right) & =p_{i}\left(0 \mid n_{i}\right) \prod_{l=1}^{k_{i}}\left[\frac{\beta_{i} \psi_{i, l-1}\left(n_{i}-1\right)}{\gamma_{i}+\beta_{i} \psi_{i, l}\left(n_{i}-1\right)}\right.
\end{aligned}
$$

and

$$
p_{i}\left(0 \mid n_{i}\right)=\left[1+\sum_{k_{i}=1}^{\infty} \prod_{l=1}^{k_{i}}\left[\frac{\beta_{i} \psi_{i, l-1}\left(n_{i}-1\right)}{\gamma_{i}+\beta_{i} \psi_{i, l}\left(n_{i}-1\right)}\right]^{-1} .\right.
$$

where we have used the fact that the conditional probabilities sum to one.

Proof The proof is straightforward, and is based on substituting (74) in the equations (73). Note also that $p_{i}(0 \mid 0)=1$.

Finally, we can write the steady-state solution under the ABA when bids and sales are frequent using (74) as:

$$
\begin{aligned}
p(n, k) & \approx p(k \mid n) p(n) \\
& \approx \prod_{i=1}^{N} \frac{e^{-U_{i}}}{U_{i}} \frac{U_{i}^{n_{i}}}{\left(n_{i}-1\right) !} p\left(0 \mid n_{i}\right) \prod_{l=1}^{k_{i}}\left[\frac{\beta_{i} \psi_{i, l-1}\left(n_{i}-1\right)}{\gamma_{i}+\beta_{i} \psi_{i, l}\left(n_{i}-1\right)}\right.
\end{aligned}
$$

An important property of these approximate solutions is that they have "product form". This means that once we have solved the traffic equations (62) which allow us to compute the rate at which bidders arrive to each of the auctions, we can obtain the marginal probability distributions for any of the auction centres separately, while the overall joint probability distribution is obtained from the product of thes emarginal distributions.

\section{CONCLUSIONS AND FURTHER WORK}

Computerised auctions are common in the Internet as part of the web based economy, and we may imagine that this trend will extend to a large part of the world's economy. Various enterprises may then be conducting their business based on a networked system of automated and networked purchasing and sales centres which will be dealing in goods, parts for manufacturing industries, transportation services and other services that enterprises may need. 
In this paper we consider auctions with "automated" bidders and sellers, where bidders arrive to auctions according to some random process, and where the time it takes a seller to make a decision is also modelled as a random variable. We analyse such systems to obtain closed form expressions for their steady state behaviour as a function of different system paramaters. In particular we consider metrics such as the sale price reached by a good in an auction, and the income per unit time that the seller obtains from repeated sales of the same good to differet buyers. We also consider the impact of the time that it takes a seller to reach its decision on the metrics of interest.

After considering a single auction, we turn to a network of $N$ auctions where buyers can circulate among a set of auctions. We suggest a mathematical model for such a system which includes both the number of buyers and the price attained by the good, at each of the $N$ auctions. An approximate analytical solution is obtained under the assumption that buyers are quite active, so that their bidding rates are significantly higher than the rate at which they move among the different auctions. It is seen that this analytical solution has "product form" which allows us to compute the probabilistic properties of each of the individual auctions separately, and then obtain the joint probability distribution for the number of bidders and the value attained by a good, from these separately calculated marginal distributions.

Some aspects concerning rationality and preferences expressed by bidders are also modelled in the case when $N$ auctions operate the same network, as discussed in Section 4. Thus the rate $\mu_{i}$ at which a bidder leaves one auction $i$ can depend on the auction itself when, for instance, some auctions are less attractive than others. Similarly, newly arriving bidders may initially choose some auction $i$ more or less frequently than other auctions through the specific arrival rate $\gamma_{i}$; thus if $\gamma=\sum_{i=1}^{N}$, the quantity $\gamma_{i} / \gamma$ is the probability that a newly arriving bidder first chooses auction $i$. The probabilities $P(i, j)$ express the fact that bidders may have some preferences in the way they successively visit different auctions, and they represent the preferences observed about the bidders' collective behaviour. For instance, certain auctions may have a higher quality level for the goods they sell, or (as is discussed in Section 4.1), the bidders may go to auctions that are busier or less busy, or to those where the sale price is generally lower; these are all instances of rational behaviour which can be modeled in the context we describe. Also the probabilities $P(i, j)$ may depend on the congestion observed at different auction centers, in which case we might take:

$$
P(i, j)=c \frac{1 /<n_{i}>}{\sum_{i=1}^{N} 1 /<n_{i}>}, 0<c<1,
$$

which represents the situation where bidders may tend to visit auctions which are less busy, as in the "sensible decision" algorithms studied in [Gelenbe 2003]. Similarly, we may consider cases where bidders are more likely to go to auctions where prices are lowetr. Of course exactly the opposite may also happen, when buyers are attracted by the crowds they see, creating even more congestion and competition for themselves, or when they are attracted to more expensive goods which are perceived to be of better quality.

We also allow distinct "values" to be attributed to goods at different auctions in the networked case, representing the fact that the formation by the bidder of an opinion about value may depend on where the bidder is. In this paper we do not 
examine optimum rules of bidder behaviour, but optimisation studies can indeed use the analysis that we present as a starting point as we have done in several examples that are given in the text.

Many interesting questions and generalisations can be considered and will be the subject of future work. First of all, the analysis we have presented leads naturally to problems of optimisation and estimation. One can study how the different system parameters, such as arrival rates of bids or decision rates of sellers, can be rationally used to optimise the benefit of the different parties involved. In the networked case, future work can study how prices evolve when buyers are attracted by lower prices, or how value changes when value formation results from observed prices. Furthermore one can study how decisions can be taken when such parameters are observed and estimated rather than known in advance.

More broadly speaking, future work will investigate how buyers' interests can be best served by examining the opportunities offered by the large number of auctions which populate the Internet, and how the buyers can automatically navigate through these auctions in order to maximise their benefit.

\section{REFERENCES}

Chow, Y. S., Moriguti, S., Robbins, H., And Samuels, S. M. 1964. Optimal selection based on relative rank (the secretary problem). Israel J. Math. 2, 81-90.

Das, N. R., Jennings, N. R., And Parkes, D. C. 2003. Computational mechanism design: A call to arms. IEEE Intelligent Systems, 40-47.

David, E., Rogers, A., Schiff, J., Kraus, S., And Jennings, N. R. 2005. Optimal design of english auctions with discrete bid levels. Proc. of 6th ACM Conference on Electronic Commerce (EC'05), 98-107.

Finch, S. R. 2003. Optimal stopping constants. Mathematical Constants, 361-363.

Gelenbe, E. 2003. Sensible decisions based on qos. Computational Management Science 1, 1, $1-14$.

Guo, X. 2002. An optimal strategy for sellers in an online auction. ACM Trans. Internet Tech. 2, 1, 1-13.

Hajiaghayi, M. T., Kleinberg, R., and Parkes, D. C. 2004. Adaptive limited-supply online auctions. Proc. 5th ACM Conference on Electronic Commerce, May 17-20, 71-90.

McAfee, R. P. And McMillan, J. 1987. Auctions and bidding. J. Economic Literature 25, $699-738$.

Medhi, J. 1994. Stochastic Processes, 2nd Ed. Wiley Eastern Ltd., New Delhi.

Milgrom, P. R. And Weber, R. 1982. A theory of auctions and competitive bidding. Econometrica 50, 1089-1122.

SheHory, O. Optimality and risk in purchase from multiple auctions. Vol. 2182. Springer Verlag, Lecture Volumes in Artificial Intelligence, 142-153.

Shenory, O. 2002. Optimal bidding in multiple concurrent auctions. International Journal of Cooperative Information Systems 11, 3-4, 315-327. 